\title{
Acerca de la metodología en la historia de la traducción. Caso de estudio: las traducciones entre el catalán y el rumano
}

\author{
Diana MOŢOC \\ Universidad Babeş-Bolyai \\ Rumanía
}

\begin{abstract}
Resumen: El presente artículo, tras un breve recorrido por la evolución reciente de los estudios de historia de la traducción, pretende dar cuenta de una metodología de la investigación histórica dentro de la traductología, basada en varios estudios esenciales sobre dicho tema, entre los que destacamos el trabajo de Sabio Pinilla (2006). Retomamos la aplicación de la propuesta del autor español, formulada como una serie de pasos esenciales para toda investigación histórica, mediante los datos proporcionados por nuestra investigación doctoral sobre la historia de las traducciones entre el catalán y el rumano.
\end{abstract}

Palabras clave: metodología, historia de la traducción, traducciones entre el catalán y el rumano.

\begin{abstract}
The present paper examines concisely the recent evolution of the history of translation studies, in order to explain a methodology of historical research in this specific area. More exactly, we focus on several key studies on the subject matter, among which are the Sabio Pinilla's publications (2006). In this respect, we resume the application proposed by the Spanish author, formulated as a series of essential steps for any historical research, using data provided from our Ph.D. thesis in the history of translations between Catalan and Romanian.
\end{abstract}

Keywords: methodology, history of translation, translations between Catalan and Romanian.

Los estudios de historia de la traducción proponen un enfoque de la actividad de traducción (y edición) con el objeto de crear un panorama detallado que comprenda el corpus de textos traducidos y tratan de responder a preguntas relacionadas con la elección de los textos, las motivaciones, la personalidad de los traductores, las circunstancias. En palabras de Judith Woodsworth, la historiografía de la traducción estudia "what has been translated, by whom, under what circumstances, and in what social and political context" $(1998,101)$.

Antoine Berman afirmaba, en el prólogo de su libro L'épreuve de l'étranger, que "la constitution d'une histoire de la traduction est la première tâche d'une théorie moderne de la traduction" $(1984,12)$. Y hacía hincapié en que: 
Il est impossible de séparer cette histoire de celle des langues, des cultures et des littératures - voire de celle des religions et des nations. Encore ne s'agit-il pas de tout mélanger, mais de montrer comment, à celle de la littérature, des langues, des divers échanges interculturels et interlinguistiques. (Berman 1984, 12-13)

Asimismo, el autor francés destaca el hecho de que la investigación del pasado puede abrir el conocimiento del presente:

Faire l'histoire de la traduction, c'est redécouvrir patiemment ce réseau culturel infiniment complexe et déroutant dans lequel, à chaque époque, ou dans des espaces différents, elle se trouve prise. Et faire du savoir historique ainsi obtenu une ouverture de notre présent (Berman 1984, 14).

La historiografía de la traducción -campo privilegiado en los últimos años dentro de la Traductología- se ha enriquecido considerablemente y trata la historia de la teoría, de las reflexiones traductológicas, así como de la práctica. Entre los más notables estudios en torno a la historia de la traducción y a la reflexión sobre la traducción están los de Henri van Hoof (Histoire de la traduction en Occident, 1991), Michel Ballard (De Cicéron à Benjamin. Traducteurs, traductions, réflexions, 1992), Hans Vermeer (Skizzen zu einer Geschichte der Translation, 1992), García Yebra (La traducción. Historia y teoría, 1994), Lawrence Venuti (The Translator's Imisibility: A History of Translation, 1995), Mona Baker y Kirsten Malmkjær (Routledge Encyclopedia of Translation Studies, 1998), Ruiz Casanova (Aproximación a una historia de la traducción, 2000), Jean Delisle y Judith Woodsworth (Les traducteurs dans l'histoire / Translators through history, 1995) o Jean Delisle (Potraits de traducteurs, 1999, Portraits de traductrices, 2002).

Notamos entre los títulos mencionados que se escribieron tanto historias particulares, centradas en cierta cultura o comunidad, como investigaciones que avanzan hacia una historia universal de la traducción. Quien propuso una historia mundial de la traducción por primera vez fue el profesor y traductor húngaro G. Radó, en el IV Congreso de la Federación Internacional de los Traductores (FIT), en 1963 (Woodsworth 1998, 105). Los que pusieron en obra, en cierta medida, la propuesta fueron los miembros del comité creado en 1991, bajo la coordinación de Jean Delisle. El fruto de los cincuenta historiadores de la traducción, de veinte países, se recogió en una pequeña historia, o introducción a la historia universal de la traducción, un libro que rinde un excepcional homenaje a los traductores, coordinado por Delisle y Woodsworts y publicado en 1995, en francés, 
primero, y luego en inglés, Les traducteurs dans l'histoire / Translators through History ${ }^{1}$.

No obstante, hasta el presente, escribir una historia universal exhaustiva parece más bien ilusorio. Sin embargo, se dieron $-\mathrm{y}$ se siguen dando- pasos importantes en el conocimiento del fenómeno de la traducción y de su papel a lo largo de los siglos, tras la elaboración de las ya mencionadas historias particulares. Cabe destacar asimismo los realizados en los dos espacios culturales investigados por nosotros, el catalán y el rumano. En este sentido, para el catalán, debemos citar los trabajos de historia de la traducción realizados bajo la coordinación de los investigadores Montserrat Bacardí, Joan Fontcuberta y Francesc Parcerisas, Cent Anys de Traducció a Catalunya, 1891-1990: Antologia, de 1998, de Montserrat Bacardí, Una impossibilitat possible. Trenta anys de traducció als Països Catalans (1975-2005) (2010), o los firmados por la misma: La traducció catalana sota el franquisme (2012) y Les traductores i la tradició (2013); así como la antología bilingüe de textos sobre la traducción de Gallén et al. (2000).

En Rumanía, destacamos los repertorios y los estudios coordinados por Georgiana Lungu Badea: Repertoriul traducătorilor români de limbă franceză, italiană şi spaniolă... (2005), Repertoriul traducerilor româneşti din limbile franceză, italiană şi spaniolă... (2006), Un capitol de traductologie românească. Studii de istorie a traducerii (2008). Los tres libros son fruto de la investigación traductológica emprendida en el marco del Centro de estudios ISTTRAROMTranslationes, a través del proyecto titulado Contribuţia traducerilor românești (sec. XVIII-XIX) din limbile franceză, italiană şi spaniolă la dezvoltarea limbii şi culturii române, a schimburilor culturale românooccidentale.

La investigadora firma asimismo trabajos de referencia en el campo de la historia de la traducción rumana: Scurtă istorie a traducerii. Repere traductologice (Lungu Badea 2007) y, sobre todo, Idei traductive şi metatraductive româneşti (sec. XVI-XXI) (Lungu Badea 2013), en el que reúne, desde una perspectiva argumentativa, datos históricos relativos a los comienzos traductivos rumanos, los traductores, los programas de traducción, así como sobre la pretraductología rumana, y establece una conexión explícita entre las preteorías inductivas de la traducción (siglos XVIII y XIX), las teorías lingüísticas deductivas y las ramas de la traductología actual.

En lo que concierne a la metodología de la investigación en la historia de la traducción, nos parece imprescindible retomar algunos puntos de vista que demuestran la incidencia y la actualidad de los estudios históricos en el enfoque diacrónico del fenómeno y de los

\footnotetext{
${ }^{1}$ El libro fue traducido al rumano (Traducătorii în istorie), también en equipo, bajo la coordinación de la traductóloga Lungu Badea, y publicado por la Editorial de la Universidad del Oeste de Timişoara, en 2008.
} 
flujos traductivos. En este sentido, Hurtado Albir (2004) introduce en cada una de las ramas de los Estudios de Traducción establecidas por Holmes (1972/1988) -estudios teóricos (generales y parciales), descriptivos y aplicados- una serie de variables, siendo una de ellas la dimensión histórica, que tiene su propia metodología de investigación. De hecho, son varios los autores que le han dedicado estudios y a algunos de ellos nos referiremos a continuación para dar cuenta de una metodología de la investigación histórica de la traducción.

A partir de los estudios descriptivos de la traducción y de la teoría de los polisistemas, Lambert (1992, citado por Sabio Pinilla 2006) propone la investigación de las traducciones a lo largo de la historia como modo de entender la evolución del concepto y de la realidad actual en los diferentes espacios o comunidades culturales. En este sentido el autor formula preguntas que todo historiador debe hacerse: quién, qué, dónde, para quién y cómo se traduce. Asimismo, analiza fenómenos como la no-traducción, el papel de las traducciones ${ }^{2}$ y de los traductores en la sociedad o en la comunidad lingüística.

Con objeto de definir de modo científico la forma de escribir la historia de la traducción, Jean Delisle (1997-98) destaca, a su vez, la aplicación del método de trabajo del historiador, es decir una búsqueda de fuentes fiables, la selección pertinente de los documentos, una interpretación que dé sentido a los hechos integrándolos en el contexto y la inclusión de un juicio de valor.

Un trabajo complejo que trata la metodología de la historia de la traducción es el estudio de Anthony Pym, Method in Translation History (1998). El método propuesto por Pym surge de aspectos prácticos y el autor formula cuatro principios estrechamente relacionados con las cuestiones reales de la historia de la traducción: 1) la causalidad de las traducciones, es decir ¿por qué se traduce?; 2) el traductor se sitúa en el centro de los estudios de historia de la traducción; 3) la interculturalidad tiene un papel central; 4) la prioridad del presente: la historia de la traducción debe tener el objeto de plantear, analizar y responder a las preguntas actuales (Pym 1998, ix-xi).

Pym (1998, 5-6) establece y define tres ramas en historia de la traducción: la arqueología, que respondería a las cuestiones: quién ha traducido qué, cómo, dónde, cuándo, para quién y con qué efecto; la crítica histórica, que evalúa la manera en que las traducciones llevan o no al progreso; y la explicación, que pretende detallar y justificar por qué aparece cierta traducción. Aunque inseparables, cada una de las tres ramas puede constituir un enfoque específico del objeto de estudio.

\footnotetext{
${ }^{2}$ En este contexto, queremos subrayar también el papel dinamizador del texto extranjero en la creación de una literatura nacional. Daniel-Henri Pageaux (2000) opina que es casi imposible estudiar una época literaria sin tener en cuenta la presencia y el papel de las traducciones.
} 
Sabio Pinilla es autor de un trabajo exhaustivo ("La metodología en historia de la traducción: estado de la cuestión", 2006) cuyo objetivo es presentar el estado de la cuestión (antecedentes del interés actual por la historia de la traducción, ubicación de la disciplina dentro de los Estudios de Traducción, su enseñanza, tipos de trabajos históricos), así como los problemas metodológicos de la investigación en historia de la traducción. Tras repasar la problemática y las cuestiones metodológicas planteadas por algunos de los más significativos trabajos dedicados a la historia de la traducción (Lambert, D’hulst, Delisle, Pym, Gallego Roca, Lépinette, López Alcalá), concluye con una propuesta general formulada como una serie de pasos fundamentales para toda investigación histórica (Sabio Pinilla 2006, 42-44). A continuación expondremos dichos pasos, tratando de ofrecer al mismo tiempo una muestra de su aplicación mediante los datos proporcionados por nuestra investigación doctoral sobre la historia de las traducciones entre el catalán y el rumano3.

Sabio Pinilla (2006) establece como primer paso la elección del tema, que debe incluir las hipótesis de trabajo que guíen la investigación. Es esencial que el tema esté bien delimitado en el tiempo y en el espacio, lo que permite al investigador centrarse en el objeto de estudio en profundidad. El autor detecta en este punto la deficiencia de haberse estudiado hasta ahora sobre todo textos y traductores conocidos, de haberse privilegiado la traducción literaria y las culturas europeas occidentales, de haberse excluido, añadiríamos, las periferias, lo que precisamente pretendemos hacer en nuestra tesis. Esta trata, en una primera fase, el contacto cultural a través de la traducción (traducciones), proporcionando un inventario de las traducciones de textos literarios del catalán al rumano y del rumano al catalán desde 1887 hasta el presente. Los límites cronológicos coinciden prácticamente con la primera traducción del catalán al rumano identificada (en manuscrito, ya que la primera publicada data de 1898) y el momento actual.

Tras haber formulado las hipótesis (la traducción tiene un papel catalizador para establecer los contactos entre culturas y lenguas, para el conocimiento mutuo, aun cuando, o sobre todo cuando se trata de dos culturas "menores" -al menos como área de extensión) y las cuestiones metodológicas (nuestra investigación se basa en un enfoque histórico-comparativo de la traducción), definimos la relación entre las dos culturas y lenguas, tratando, al mismo tiempo, la problemática político-lingüística de los conceptos de lenguas "mayores"/"menores", ya que el rumano y el catalán son lenguas más bien "exóticas" una para la otra y las creaciones de cada una tienen un estatuto marginal en la cultura meta. Hay una relación de "periferia-periferia", podríamos decir

3 "Traducerea, loc de întâlnire a culturilor şi limbilor catalană şi română. Studiu de caz: culturemul”, tesis doctoral, defendida en mayo 2015, en la Universidad del Oeste de Timişoara. 
en términos de la teoría del polisistema, o de "cultura dominada-cultura dominada”, en la terminología de la teoría de los bienes culturales, lo que ciertamente influenció en la actividad de traducción.

Un segundo paso mencionado por Sabio Pinilla (2006) es buscar las fuentes de información y documentos auténticos. El investigador español destaca la utilidad de los trabajos bibliográficos, de los catálogos y de los repertorios. En cuanto a lo que nos interesa, todos los tipos de fuentes mencionadas, y también el contacto directo con los traductores, editores y autores -como participantes en el proyecto de traducción literaria del catalán al rumano- nos han permitido acceder a las fuentes primarias. En la bibliografía fundamental, destacamos el trabajo de Ribera Llopis (2009) y los de Xavier Montoliu Pauli (2008, 2011), que, además de los datos proporcionados y los comentarios pertinentes, son muy significativos, ya que al ser su procedencia la tierra del Otro, ofrecen una mirada desde esta perspectiva sobre la traducción de la literatura catalana y su recepción en Rumanía. El itinerario propuesto por Ribera Llopis se refiere principalmente a las traducciones surgidas en publicaciones periódicas al final del siglo XIX y comienzos del siglo XX (años 30), material recuperado en gran parte del trabajo Bibliografia relaţiilor literaturii române cu literaturile străine (Lupu y Ştefănescu 19801985, Grigorescu, 1997-2005). Montoliu continúa la cronología, llevando la investigación hasta el presente (2008). Advertimos también el hecho de que Jana Balacciu Matei y Xavier Montoliu Pauli escriben sobre los contactos culturales y literarios rumano-catalanes del presente, siendo ellos mismos sus iniciadores y hacedores, puentes entre las dos lenguas y literaturas, como traductores (los dos) y editores (Balacciu Matei). Balacciu Matei, mediante su trabajo de traductora y editora de crítica y prologuista, de organizadora o invitada en talleres de traducción, en ferias y presentaciones de libros, pone a disposición un material amplio y vivo, un auténtico work in progress.

En su estudio, Sabio Pinilla (2006) subraya que se impone una contextualización adecuada del tema escogido, las traducciones y los traductores, debiéndose analizar el contexto social, político, económico, ideológico y cultural. La traducción es un fenómeno de intercambio cultural que supone una percepción clara, precisa $\mathrm{y}$ detallada del contexto que la genera y al mismo tiempo al que se dirige (Pageaux 2000). En nuestra investigación, el recorrido histórico previo de los géneros literarios catalanes y de los más importantes representantes de los mismos tiene el propósito de facilitar la respuesta a las preguntas: quiénes son los autores y qué textos han escogido los traductores rumanos, cuál es su contexto de producción y su lugar en el espacio cultural de origen. Al mismo tiempo, el análisis de las circunstancias histórico-políticas, ideológicas y lingüísticas revelan tanto la situación específica de la literatura catalana, como de las traducciones del catalán al rumano. Por otro lado, la presentación del 
contexto traductivo rumano ofrece y matiza la perspectiva sobre el intercambio literario entre las dos culturas mediante la traducción.

El siguiente paso de Sabio Pinilla (2006) es establecer una periodización adecuada al tema, una evolución propia basada en la cronología. En el caso de las traducciones del catalán al rumano, la investigación respeta una cronología que la historia misma de las traducciones tratadas ha impuesto. Así, la primera fase de esta aventura comienza al final del siglo XIX, un siglo en que, en pleno romanticismo y simétricamente, las dos literaturas nacen (la rumana, como literatura moderna) o renacen (la catalana, a través de la Renaixença) y finaliza por razones histórico-políticas evidentes poco después de los años 30. La preciosa difusión de las letras catalanas en Rumanía sigue, aunque más tímida y esporádicamente, solo después de los años 70 (segunda fase), cuando se hace sentir una cierta apertura del régimen comunista rumano. En el año 1993, la Editorial Univers publica la primera traducción directa del catalán de una novela, Piaţa Diamantului [La plaça del Diamant] de Mercè Rodoreda a cargo de Balacciu Matei y Montoliu Pauli. El año 1997, cuando la Editorial Meronia de Bucarest crea la colección "Biblioteca de Cultură Catalană", consagrada exclusivamente a las obras catalanas y cuyo repertorio cuenta hasta ahora con 37 títulos, abre una "edad de oro" de las traducciones del catalán al rumano, enriquecida considerablemente por las iniciativas de otras editoriales rumanas que han incluido en su oferta traducciones de la literatura catalana. En cambio, en la investigación de las traducciones del rumano al catalán no hemos utilizado periodización alguna, debido a que su historia, más reciente y menos rica, no era apropiada para ello.

El paso más consistente se refiere a la interpretación del pasado, a la evaluación del mismo, a la perspectiva crítica sobre los resultados obtenidos, partiendo de las respuestas a las preguntas ya clásicas de la investigación: quién, qué, dónde, con qué propósito, por qué, cómo o cuándo, ya que el estudio de la historia es un esfuerzo de compresión y de interpretación que debe ser útil para todos (Sabio Pinilla, 2006). El análisis de las traducciones entre el catalán y el rumano ha sido uno descriptivo y cuantitativo (exhaustivo). Hemos estudiado la actividad de traducción y de edición hasta el presente, y hemos compuesto una bibliografía detallada y un repertorio anexado, que comprende el corpus de textos traducidos, editados y puestos en circulación; obteniendo de este modo un espejo de las traducciones "producidas" realmente: escritas, editadas (o no) y re-editadas (signo de cierta resonancia). Hemos presentado los autores, los libros traducidos, los traductores, las editoriales y las demás instituciones, que participan en la actividad de traducción y su recepción en la cultura meta o la determinan 4 . Los datos matizan, entre otras cosas, la respuesta a la

4 Sobre los intercambios entre la literatura rumana y la literatura catalana, v. la revista VISAT del PEN Català: http://www.visat.cat/articles/cat/70/quatre- 
pregunta por qué se ha traducido (aunque tarde) entre las dos culturas y lenguas, es decir, si ha sido una demanda de la cultura meta o una oferta de la parte de la cultura original.

Queremos subrayar en este punto la necesidad de la aplicación de la estadística en la historia de la traducción, es decir de un "método exacto de investigación", que contribuya a la "elucidación del problema de las traducciones", como observa Cornea $(1966,38)$ en el estudio emprendido acerca de las traducciones laicas entre 1780-186o en Rumanía. Dice él:

[...] la meta impone los medios, ya que es evidente que para obtener una perspectiva absolutamente objetiva sobre el fenómeno de las traducciones, más allá de toda intuición arbitraria y de toda inferencia ensayística de lo particular a lo general, ninguna vía parece más indicada que la de inventariar la totalidad de los casos y clasificar sistemáticamente los resultados. (Cornea 1966, 39)

Cornea (1966) toma en cuenta varios considerantes en la aplicación de este método de investigación en la historia literaria (de la traducción literaria), entre los que mencionamos que el análisis estadístico se puede aplicar en el caso en que: a) nos ocupemos de sistemas relativamente aislados; b) que las unidades para sumar se puedan definir rigurosamente, o que tengan una existencia autónoma y sean conmensurables; c) que dispongamos del inventario completo de los casos considerados o de una serie de muestras suficientes (1966, 45). Este es precisamente el caso del estudio de las traducciones del catalán al rumano y del rumano al catalán.

Los resultados estadísticos de la investigación y las conclusiones que se desprenden de los mismos son evidentes, "ya que allí donde hablan las cifras, las palabras sobran” (Cornea 1966, 47). Podemos notar, en primer lugar, una evidente asimetría entre las traducciones de la literatura catalana al rumano (que suman un número de 108 traducciones publicadas) y las de la literatura rumana en catalán (9 traducciones). Observamos, asimismo, que las últimas fueron realizadas después de 1990, que en su mayoría son traducciones de poesía y que sólo 6 del número total fueron publicadas en volumen. Por consiguiente, la apertura de los rumanos hacia las letras catalanas no se corresponde con una presencia similar de los autores rumanos en Cataluña, a pesar de su política de emancipación lingüística y cultural que incluye la traducción desde y hacia el catalán. Entre las razones identificadas por Montoliu Pauli (2008, 114), mencionamos el número escaso de traductores del rumano al catalán, el desconocimiento del canon literario rumano, y la preferencia de los editores catalanes para

apuntsnbspsobre-les-relacionsnbspentre-la-literatura-romanesa-i-lacatalana.html. 
publicar traducciones de las culturas centrales financiadas por el país de origen.

Otra asimetría llena de significaciones destaca en el volumen de las traducciones del catalán al rumano. Si desde el comienzo del siglo XX hasta los años 30 se manifiesta un cierto interés de los intelectuales rumanos hacia las letras catalanas, materializado principalmente en traducciones de poesía publicadas en periódicos (31 títulos), en el contexto de la guerra civil y, luego, del franquismo, así como durante el periodo de la más severa censura comunista en Rumanía (1930-1972), no hemos identificado ninguna traducción de la lengua catalana. En cambio, observamos un crecimiento significativo del número de traducciones después de los años 90, tras la salida de la cultura rumana del régimen totalitario y en el contexto del esfuerzo de recuperación de las producciones literarias, sobre todo de las culturas hegemónicas, aunque también de las menos conocidas. Por consiguiente, desde 1990 hasta el presente ( 25 años) se han traducido y publicado 64 títulos, comparado con 44 en todo el periodo anterior (92 años). Además, la gran mayoría se han publicado en volumen (6o) y, más de la mitad (37) en la editorial Meronia, en la colección "Biblioteca de Cultură Catalană”.

El análisis por géneros destaca, en el primer periodo, la traducción de poesía en detrimento de la prosa, ya que se prestaba, sobre todo en la primera mitad del siglo XX, a la intención de ofrecer una serie de muestras de la literatura catalana para el público rumano (Iorga publica en 1930 algunas "muestras de poesía catalana", medieval, moderna, popular; y Papahagi, en 1972, selecciona, aunque de un solo periodo literario, un conjunto concentrado, pero rigurosamente ilustrativo). La novela, en cambio, suponía una empresa de envergadura, y tal vez un mejor conocimiento de los elementos culturales de la lengua de origen. Solo tras los años 90, esta cobra cada vez mayor importancia: si hasta 1990 se tradujeron sólo tres novelas (dos probablemente mediante el francés), en los últimos años las editoriales rumanas se han decantado por este género (39 títulos).

Más allá de los factores histórico-políticos mencionados, creemos que una causa de estas asimetrías y desequilibrios es la falta de un programa coherente de promoción de cada una de las literaturas en el espacio de la otra y una visión a largo plazo. Observamos en este sentido la intención de la Editorial Meronia mediante la creación de la colección "Biblioteca de Cultură Catalană", gracias a la iniciativa ejemplar y al esfuerzo de la traductora Balacciu Matei. De hecho, las iniciativas, tanto en los tiempos pasados como en los presentes, han sido de los traductores, animados por su pasión de conocer al Otro, la literatura del Otro, y por el afán de compartirla (traducida) en su sociedad. Si para Iorga la traducción significó una misión cultural nacional, para Montoliu Pauli "las fronteras son caminos, vías de comunicación” (Balacciu Matei 2008). Al negarles la función que 
siempre han ejercido, estas llegan a ser fluidas gracias a la translación (en el sentido etimológico de la tra-ducción, "llevar entre").

En una acepción comúnmente admitida, el traductor es un profesional responsable que tiene una misión de cooperación intercultural. Por ello, su personalidad, como mediador lingüístico y cultural, y como iniciador de la traducción, es reveladora para el encuentro traductivo catalán-rumano, especialmente en el caso de la traductora y editora Balacciu Matei. El traductor, prescriptor de la literatura que traduce, es asimismo, en el caso concreto de nuestra investigación, el constructor de un paradigma de la literatura catalana en rumano. En las dos primeras etapas de la traducción del catalán al rumano, los traductores identificados son por excelencia escritores, personalidades culturales, políglotas, que hablan varias lenguas románicas (Nicolae Iorga, Marian Papahagi, Virgil Ani etc.), o hispanistas (Al. Popescu-Telega o Victor Ivanovici), con cierta iniciación en el catalán 5 , que solo tradujeron un número reducido de poemas, a veces un único libro, tratándose más bien de un afán de descubrirle al lector rumano la literatura catalana, que de una profesionalización del trabajo. Esta llegará después de los años 90, cuando los que firman las traducciones son catalanohablantes, mediante estudio particular (Balacciu Matei), y también institucional, tras la creación del lectorado de catalán en la Universidad de Bucarest en 1992. En cuanto al traductor Montoliu Pauli, primer lector de catalán de la Universidad de Bucarest, ha enseñado a muchos rumanos su lengua y "les ha hecho conocer, admirar y amar su cultura", pero también "ha recorrido con la misma pasión el camino de vuelta" (Balacciu Matei 2009).

Interesado por todo y asimilando todo lo que es rumano, desde el paisaje hasta la vida cotidiana, la literatura y el arte, pasando por la lengua rumana que habla de manera impecable, llegó a ser, al volver a casa, un embajador sin descanso del mundo rumano. (Balacciu Matei $\left.2009^{6}\right)$

Además de las muchas traducciones de poemas en revistas y artículos sobre la recepción de la literatura rumana en el sistema literario catalán, una de las más notables contribuciones del traductor catalán al conocimiento de la literatura rumana es haber traducido, junto con Corina Oproae, una selección de la poesía de Marin Sorescu, Per entre els dies. Antologia (2013), así como el libro ensayístico La cinquena impossibilitat (2014), de Norman Manea.

El papel del traductor, con su competencia bilingüe y bicultural, es aun más dinamizador como intermediario en la

5 El catalán, al ser cercano a estas lenguas, y también al rumano, permite una fácil comprensión.

${ }^{6}$ Edición en línea, sin número de páginas. 
comunicación intercultural, al realizar una transferencia entre enclaves lingüísticos, podríamos decir, al límite, de las lenguas catalana y rumana. Por ello, sus iniciativas, quizás más que las circunstancias político-históricas y lingüísticas, nos pueden revelar por qué tenemos una historia (aunque reciente) de las traducciones entre el catalán y el rumano, es decir por qué tenemos (o en qué medida tenemos) una literatura traducida de cada una estas lenguas en la otra.

Para concluir, destacamos que la metodología aplicada nos ha permitido demostrar que la traducción es una mediación cultural esencial. Si la posición central de una cultura (dominante) favorece las traducciones, a estas les corresponde tanto más el papel fundamental de establecer un vínculo directo (a veces el único) entre culturas y lenguas más bien periféricas o menos conocidas. Más aun, la traducción puede convertirse en el único lugar de encuentro entre dos culturas y lenguas, o por lo menos en un lugar privilegiado, tal y como sucede con las traducciones entre el catalán y el rumano.

En definitiva, el conocimiento histórico debería poner a nuestra disposición un instrumento de investigación para la evaluación del pasado; y por consiguiente, un último paso en opinión de Sabio Pinilla (2006), es realizar una posible revisión de la historia con la proposición de nuevos campos de investigación. Como segunda conclusión (prospectiva), diremos que la historia de las traducciones entre el catalán y el rumano ha abierto una nueva perspectiva de investigación que matiza la respuesta a la pregunta sobre cómo se ha traducido, y que destaca el análisis comparativo bilingüe (catalán-rumano) de la traducción de los culturemas, al ser estos el lugar de encuentro en el tejido mismo del texto de dos culturas a través del proceso y el resultado de la traducción. Por el análisis de la transferencia de los elementos culturales podemos conocer la relación entre dos lenguas y literaturas mediante reflexiones sobre el proceso traductor en sí.

\section{Referencias bibliográficas}

Balacciu Matei, Jana, Xavier Montoliu Pauli. "Traduccions catalano-rumanes: antecedents i present del projecte editorial Meronia”. Revista de filología románica, 20, 2003: 163-170.

Balacciu Matei, Jana. "Xavier Montoliu Pauli: „Grenzen sind Straßen, Graniţele sunt drumuri!”/ de Jana Balacciu Matei”. Ziarul de duminică, 2012. URL: http://www.zf.ro/ziarul-de-duminica

Berman, Antoine. L'épreuve de l'étranger. Culture et traduction dans l'Allemagne romantique. Paris, Gallimard, 1984.

Cornea, Paul. "Traduceri şi traducători în prima jumătate a secolului al XIXlea”. De la Alecsandri la Eminescu. Aspecte - figuri - idei. Bucureşti, Editura pentru Literatură, 1966: 38-76.

Delisle, Jean. "Réflexions sur 1'historiographie de la traduction et ses exigences scientifiques”. Équivalences, 1997-98, 26 (2), 27 (1) : 21-43. 
Delisle, Jean, Woodsworth, Judith (eds.). Traducătorii în istorie. Traducere coordonată de Georgiana Lungu Badea. Timişoara, Editura Universităţii de Vest, 2008.

Dumitrescu, Domniţa. "Ecos catalanes en la cultura rumana". Cahiers roumains d'études littéraires, 1, 1979: 21-29.

Grigorescu, Dan. Bibliografia relaţiilor literaturii române cu literaturile străine în periodice 1919-1944. Bucureşti, Saeculum, 1997-2005.

Hurtado Albir, Amparo. Traducción y Traductología. Introducción a la Traductología. Madrid: Cátedra, 2004.

Iorga, Nicolae. O mică ţară latină: Catalonia şi exposiţia din 1929. Note de drum şi conferinţe. Bucureşti, Editura Casei Şcoalelor, 1930.

Ivanovici, Victor. "Resurecţie catalană”. Secolul XX, 180, 1, 1976: 47-49.

Kohn, János. "Romanian Tradition”. Mona Baker y Kirsten Malmkjær (eds.), Routledge Encyclopedia of Translation Studies. London/New York, Routledge, 1998, pp. 533-541.

Lungu-Badea, Georgiana. Teoria culturemelor, teoría traducerii. Timişoara, Editura Universităţii de Vest, 2004.

Lungu-Badea, Georgiana. Tendinţe în cercetarea traductologică. Timişoara, Editura Universităţii de Vest, 2005.

Lungu-Badea, Georgiana. Repertoriul traducătorilor români de limba franceză, italiană, spaniolă din secolele al XVIIIlea şi al XIX-lea. Studii de istoria traducerii (I), 2006a.

Lungu-Badea, Georgiana. Repertoriul traducerilor româneşti din limbile franceză, italiană, spaniolă din secolele al XVIII-lea şi al XIX-lea. Studii de istoria traducerii (II), 2006b.

Lungu-Badea, Georgiana. Scurtă istorie a traducerii. Repere traductologice, Timişoara. Editura Universităţii de Vest, 2007.

Lungu-Badea, Georgiana (ed.). Un capitol de traductologie românească. Studii de istorie a traducerii. Timişoara, Editura Universităţii de Vest, 2008.

Lungu-Badea, Georgiana. Idei şi metaidei traductive româneşti (secolele XVIXXI). Timişoara, Editura Universităţii de Vest, 2013.

Lupu, Ioan, Ştefănescu, Cornelia. Bibliografia relaţiilor literaturii române cu literaturile străine în periodice 1859-1918. Bucureşti, Editura Academiei Republicii Socialiste Române, 1980-1985.

Montoliu Pauli, Xavier. "Literatura romanesa i literatura catalana: quan el desafiament es diu traducció”. Quaderns. Revista de traducció, 15, 2008: 103117.

Montoliu Pauli, Xavier. “¿Qui acompaña qui? De veus literàries catalanes en romanès”. Serra d'Or, desembre, 2011: 67-70.

Montoliu Pauli, Xavier, Moţoc, Diana. "Quatre apunts sobre les relacions entre la literatura romanesa i catalana”. Visat, La revista digital de literatura i traducció del Pen Català, núm. 15, abril 2013. URL: http://www.visat.cat/articles/esp/ 70/quatre-apuntsnbspsobre-les-relacionsnbspentre-la-literatura-romanesa-i-lacatalana.html

Moţoc, Diana. „Sur la (les) méthode(s) dans l'étude historique - comparative des traductions. Le cas des traductions du catalan en Roumanie”, in Actes du 
Colloque international de Traductologie et Traduction - De la méthode en traduction et en traductologie. Timişoara, Eurostampa, 2013: 133-144.

Moţoc, Diana. "Les traductions entre le catalan et le roumain : l'histoire d'une rencontre culturelle récente". Magda Jeanrenaud, Julia Richter, Larisa

Schippel (eds.), Traducerile au de cuget să îmblînzească obiceiurile..., Rumänische Übersetzungsgeschichte - Prozesse. Produkte. Akteure, Berlin, Frank \& Timme, 2014: 293-307.

Pageaux, Daniel-Henry. Literatura generală şi comparată. Traducere de Lidia Bodea. Bucureşti, Editura Polirom, 2000.

Pym, Antony. Method in Translation History. Manchester, St. Jerome, 1998.

Ribera Llopis, Joan M. "Notícies i traduccions catalanoromaneses al llindar del nou-cents. Material de treball”. Actes del catorzè col-loqui internacional de llengua i literatura catalanes, Publicacions de l'Abadia de Montserrat, 2009: 383-392.

Sabio Pinilla, José Antonio. "La metodología en la historia de la traducción: estado de questión”. Sendebar. Revista de traducción e interpretación, vol. 17, 2006: 21-47.

Steiner, George. Après Babel. Une poétique du dire et de la traduction. Traduit par Lucienne Lotringer. Paris, Albin Michel, 1978.

Vega, Miguel Angel. Textos clásicos de Teoría de la traducción. Madrid, Cátedra, 1994.

Vleja, Victoria Luminiţa. "Despre începuturile tălmăcirilor hispanice în limba română”. Studia in honorem magistri Vasile Frăţilă, Editura Universităţii de Vest, 2005: 553-559.

Woodsworth, Judith. "History of Translation". Mona Baker şi Kirsten Malmkjær (eds.), Routledge Encyclopedia of Translation Studies. London/New York, Routledge, 1998: 100-105. 